Article

\title{
Flexible PZT Thin Film Tactile Sensor for Biomedical Monitoring
}

\section{Hong-Jie Tseng ${ }^{1}$, Wei-Cheng Tian ${ }^{2,3,4}, *$ and Wen-Jong Wu ${ }^{1, *}$}

1 Department of Engineering Science and Ocean Engineering, National Taiwan University, Taipei 10617, Taiwan; E-Mail: hjtseng@mems.iam.ntu.edu.tw

2 Department of Electrical Engineering, National Taiwan University, Taipei 10617, Taiwan

3 Graduate Institute of Electronics Engineering, National Taiwan University, Taipei 10617, Taiwan

4 Graduate Institute of Biomedical Electronics and Bioinformatics, National Taiwan University, Taipei 10617, Taiwan

* Authors to whom correspondence should be addressed; E-Mails: wctian@cc.ee.ntu.edu.tw (W.-C.T.); wjwu@ntu.edu.tw (W.-J.W.); Tel.: +886-2-3366-9852 (W.-C.T.); +886-2-3366-5764 (W.-J.W.); Fax: +886-2-2367-1909 (W.-C.T.); +886-2-3366-5724 (W.-J.W.).

Received: 16 February 2013; in revised form: 15 April 2013 / Accepted: 18 April 2013 / Published: 25 April 2013

\begin{abstract}
This paper presents the development of tactile sensors using the sol-gel process to deposit a PZT thin-film from $250 \mathrm{~nm}$ to $1 \mu \mathrm{m}$ on a flexible stainless steel substrate. The PZT thin-film tactile sensor can be used to measure human pulses from several areas, including carotid, brachial, finger, ankle, radial artery, and the apical region. Flexible PZT tactile sensors can overcome the diverse topology of various human regions and sense the corresponding signals from human bodies. The measured arterial pulse waveform can be used to diagnose hypertension and cardiac failure in patients. The proposed sensors have several advantages, such as flexibility, reliability, high strain, low cost, simple fabrication, and low temperature processing. The PZT thin-film deposition process includes a pyrolysis process at $150{ }^{\circ} \mathrm{C} / 500{ }^{\circ} \mathrm{C}$ for $10 / 5 \mathrm{~min}$, followed by an annealing process at $650{ }^{\circ} \mathrm{C}$ for $10 \mathrm{~min}$. Finally, the consistent pulse wave velocity (PWV) was demonstrated based on human pulse measurements from apical to radial, brachial to radial, and radial to ankle. It is characterized that the sensitivity of our PZT-based tactile sensor was approximately $0.798 \mathrm{mV} / \mathrm{g}$.
\end{abstract}

Keywords: PZT thin-film; sol-gel; sensor; biomedical 


\section{Introduction}

Capacitive-based, piezoresistive-based, and piezoelectric-based sensors are commonly used for tactile sensing. A flexible membrane and gap are typically included in a capacitive-based sensor, which can be widely used for applications in mobile robot contact force arrays [1], pressure sensors [2,3], proximity sensors [4], and tactile sensing arrays [5]. The applied pressure of piezoresistive-based tactile sensors [6] results in resistance changes and can be used for force sensors [7], pressure sensors [8], and tactile sensors [9]. However, capacitive-based tactile sensors typically require high voltage operation, whereas piezoresistive-based tactile sensors encounter signal drift caused by temperature changes. Therefore, piezoelectric-based sensors were chosen to examine tactile sensing applications.

Among various piezoelectric-based materials, lead zirconium titanate (PZT) thin-film is an excellent ferroelectric material for tactile sensor applications. PZT-based sensors have several advantages, such as high sensitivity, wide frequency bandwidth, and fast response. Thus, these sensors can be widely used for micro-electromechanical systems (MEMS) applications, such as in the areas of transducers [10], micromirrors [11,12], switches [13], gas sensors [14], pyroelectric sensors [15,16], energy harvesting devices [17-19], and tactile sensors [20]. Tactile sensors fabricated using MEMS offer the advantages of small size, mature technologies, and low cost processing. PZT thin-films fabricated using the sol-gel method provides the advantages of easy processing, low annealing temperature, and excellent piezoelectric characteristics. Accurate $\mathrm{Zr} / \mathrm{Ti}$ element composition can be controlled in the sol-gel deposition process; thus, a composite material with a high ferroelectric property (Zr/Ti with a ratio of 52:48) can be obtained.

Various sensors, such as piezoelectric-based sensors [21-25], optical sensors [26-29], and laser Doppler [30] sensors have been used for measurements of human body pulses at various artery regions. For piezoelectric-based tactile sensors, the mechanical energy can be transferred to electrical energy using an applied pressure, and the sensors have the advantages of high sensitivity, improved hysteresis, excellent repeatability, and high durability. In addition, flexible materials, such as aluminium nitride (AIN), lead-lanthanum-zirconate-titanate (PLZT), and polyvinylidene difluoride (PVDF) can be used for piezoelectric-based sensing applications.

We developed a novel sol-gel process to fabricate the PZT thin-film on a flexible stainless steel substrate. The proposed process has the advantages of simple fabrication with a lower cost for various applications. The fabricated tactile sensors have high sensitivity and fast strain response, and the flexible substrate enabled rugged human body monitoring, such as sensing human pulses at various topologies of several human artery areas. For example, the topology of the carotid artery area is flat, whereas the finger is curved. The PZT-based tactile sensors fabricated using the novel sol-gel process on a flexible stainless steel substrate can be used as rugged human pulse sensors with high performance. Future work application, the PZT thin film tactile sensor on stainless steel substrate and can be applied the structure health monitoring such as the bridge [31] or engineering structure [32]. In addition, the flexible PZT tactile sensor to apply energy harvesting [33], large area tactile sensors array [34], robot hard [5], touch sensor [35], pressure sensor [36] and fingerprint [37]. 


\section{Experimental Section}

\subsection{Solution Based Process for PZT Thin Film Formation}

During the sol-gel deposition process, the PZT solution was spun on the stainless steel substrate. Two chemical reactions were performed during the sol-gel process, including hydrolysis and condensation reactions. The chemical reaction equations [38] of the hydrolysis reactions are shown in Equation (1), whereas the chemical reaction equations of the condensation reactions are shown in Equations (2) and (3):

$$
\begin{gathered}
\mathrm{M}(\mathrm{OR})+\mathrm{H}_{2} \mathrm{O} \rightarrow \mathrm{M}(\mathrm{OH})+\mathrm{ROH} \\
\mathrm{M}(\mathrm{OH})+\mathrm{M}(\mathrm{OH}) \rightarrow \mathrm{M}-\mathrm{O}-\mathrm{M}+\mathrm{H}_{2} \mathrm{O} \\
\mathrm{M}(\mathrm{OH})+\mathrm{M}(\mathrm{OR}) \rightarrow \mathrm{M}-\mathrm{O}-\mathrm{M}+\mathrm{ROH}
\end{gathered}
$$

The PZT solution used to prepare the PZT thin-film in the sol-gel process consisted of lead acetate, zirconium n-prop-oxide, and titanium iso-propoxide, and the molar ratio of these compounds was 1.1:0.52:0.48. Organic solvents, such as acetic acid, lactic acid, glycerol, and ethylene glycol can be added to the mixed powders to prepare the PZT mixing solution.

The preparation steps of the PZT mixing solution are shown in Figure 1. First, Solution A was prepared by mixing the zirconium n-prop-oxide and titanium iso-propoxideat at room temperature for $30 \mathrm{~min}$. Second, Solution B was prepared by mixing lead acetate and acetic acid at $110{ }^{\circ} \mathrm{C}$ for $5 \mathrm{~min}$. Next, Solution A was mixed with Solution B for $15 \mathrm{~min}$. The mixture of Solutions A and B was subsequently mixed with DI water for $15 \mathrm{~min}$, followed by mixing with lactic acid for $15 \mathrm{~min}$ and glycerol/ethylene glycol for $15 \mathrm{~min}$ to obtain the final solution with a concentration of $1.9 \mathrm{M}$.

Figure 1. Preparation process of PZT solution Device.

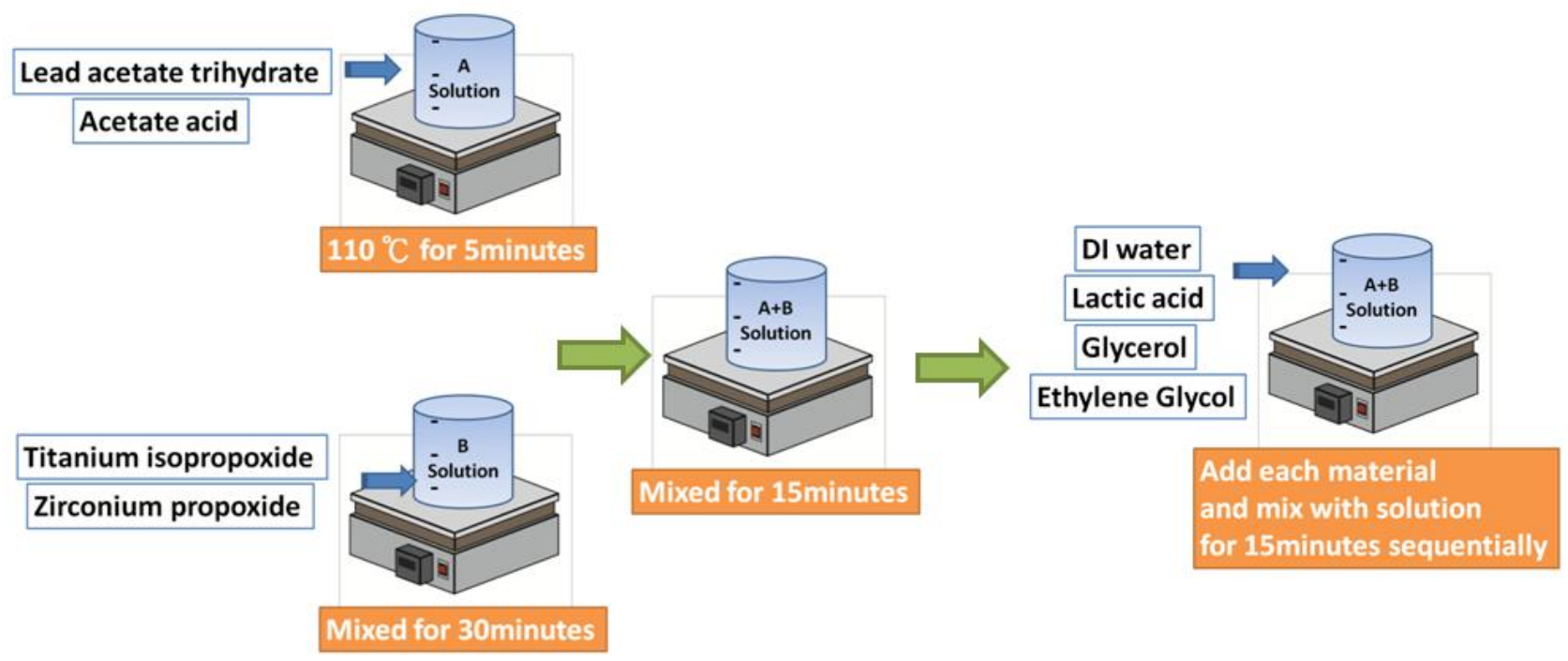

\subsection{Device Fabrication}

The $100 \mu \mathrm{m}$-thick stainless steel substrate for the tactile sensor was cleaned using ultrasound in acetone, isopropyl alcohol, and DI water for $10 \mathrm{~min}$. Next, the PZT sol-gel was spin-coated on the 
stainless steel substrate followed by the pyrolysis processes at temperatures of $150{ }^{\circ} \mathrm{C}$ and $500{ }^{\circ} \mathrm{C}$ for $10 \mathrm{~min}$ and $5 \mathrm{~min}$, respectively. After the pyrolysis process, the thin-film was annealed at $650{ }^{\circ} \mathrm{C}$ for 10 min. Finally, the Au, which served as the top electrode, was deposited using a shadow mask and a metal sputter on the PZT thin-film to complete the sensor fabrication process. A poling voltage of approximately $50 \mathrm{~V}$ was applied to the PZT film to activate the PZT tactile sensor. Finally, the polyimide tape was used to cover the sides and corners of the PZT thin film sensor and to secure the sensor on the plastic element. The overall fabrication and packaging process were shown in Figure 2(a). The packaged sensor was shown in Figure 2(b). The detailed geometries and dimensions of the different parts of tactile sensor were show in Figure 2(c).

Figure 2. (a) Fabrication process of PZT flexible tactile sensors, (b) a packaged PZT thin film tactile sensor, (c) detailed geometries and dimensions of the different parts of tactile sensor.

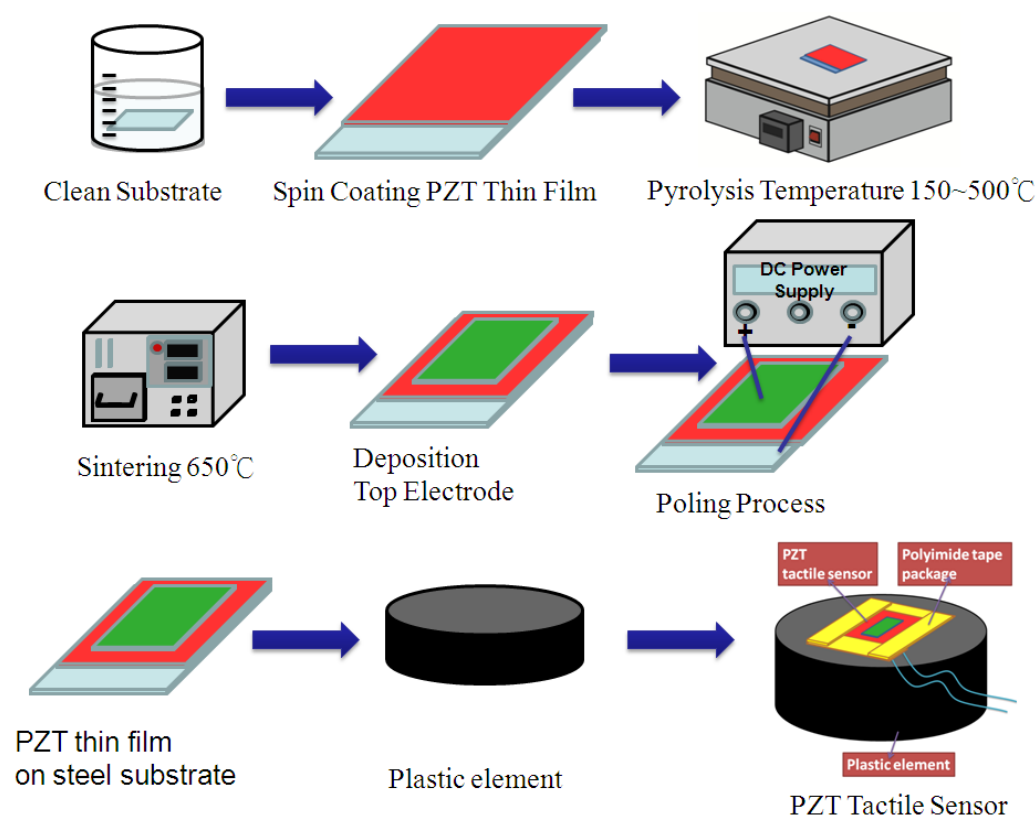

(a)

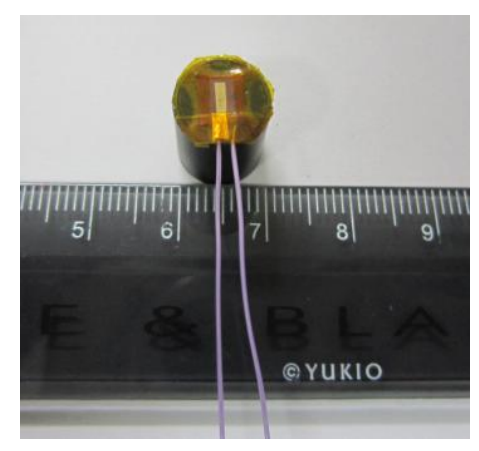

(b)

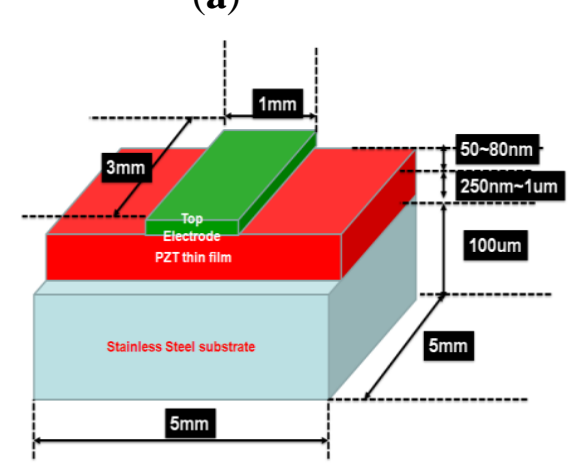

(c)

\subsection{Tactile Sensors Experimental Set-Up}

As shown in Figure 3, the PZT-based tactile sensors were used to measure human pulses. The signals from the sensor were conditioned using the self-built testing system, including a charge amplifier (Measurement Specialties, piezo film lab amplifier, Hampton, VA, USA) and an oscilloscope (Agilent technology, Digital oscilloscope Agilent Infiniium DSO9404A, Santa Clara, CA, USA). Various 
regions from the human body were chosen for pulse measurements, such as the carotid artery, the brachial artery, the finger, the ankle artery, and heartbeats. The tactile sensor was connected to the charge amplifier to magnify the sensor signal, and a filter was used to remove the undesirable signals outside the targeted frequency $(1-10 \mathrm{~Hz})$. Finally, the conditioned pulse signals were observed and recorded using an oscilloscope.

Figure 3. Experimental set-up for PZT flexible tactile sensor characterizations.

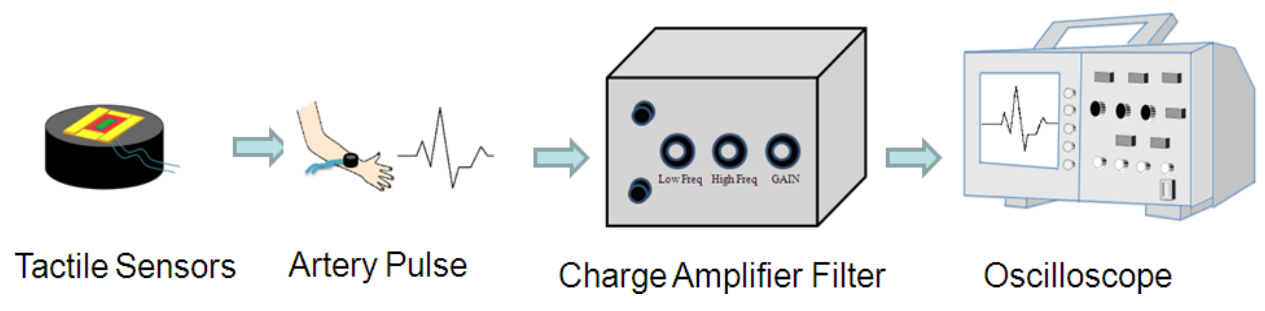

\section{Results and Discussion}

\subsection{Characterizations of PZT Films and Tactile Sensors}

\subsubsection{Surface Morphology and Crystal Orientation of PZT Thin Films}

Scanning electron microscopy (Hitachi, S4800, Tokyo, Japan) and an X-ray diffraction system (BRUKER, D8-SSS, Berlin, Germany) were used to characterize the fabrication results. Single and multi-layer deposition of the PZT thin-film on the flexible stainless steel substrate using the sol-gel process are shown in Figure 4(a-c). A PZT thin-film layer of $300 \mathrm{~nm}$ was obtained using a single spin-coating process. A $900 \mathrm{~nm}$ layer was obtained by spin coating the PZT thin-film three times. Uniform grains were clearly observed on the surface of the PZT thin-film on the flexible stainless steel substrate. Various major peaks at the main directions of the perovskite phase at $\langle 110\rangle,\langle 100\rangle$, and $<111>$ were observed using X-ray diffraction to measure the crystal orientation of the PZT thin film; the PZT thin-film was polycrystalline, as shown in Figure 5.

Figure 4. PZT thin films deposited from single or multilayer spin coating (a) single layer (b) double layer (c) triple layer.
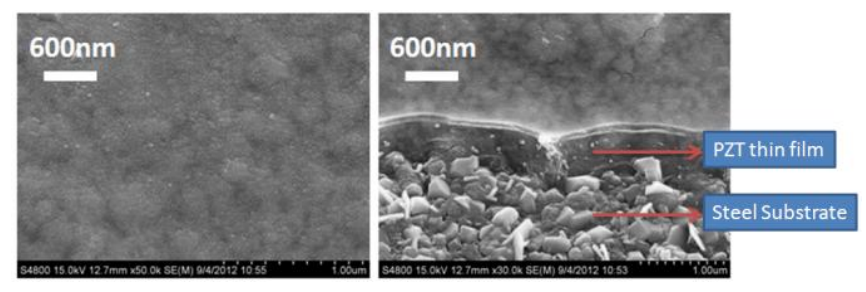

\section{(a)}

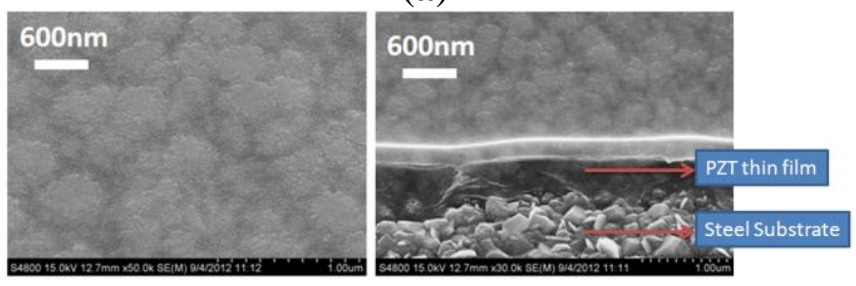

(b) 
Figure 4. Cont.

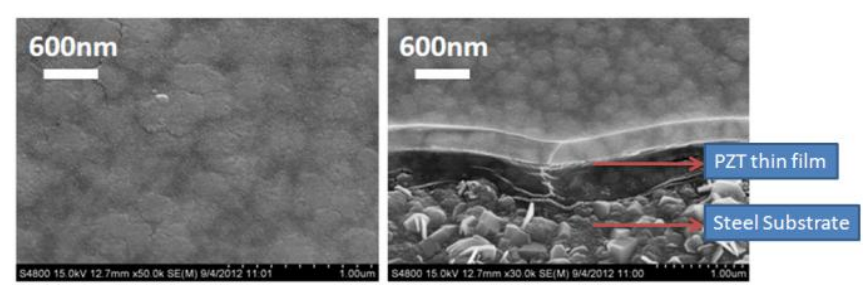

(c)

Figure 5. A PZT thin film crystal orientation on flexible stainless steel substrate.

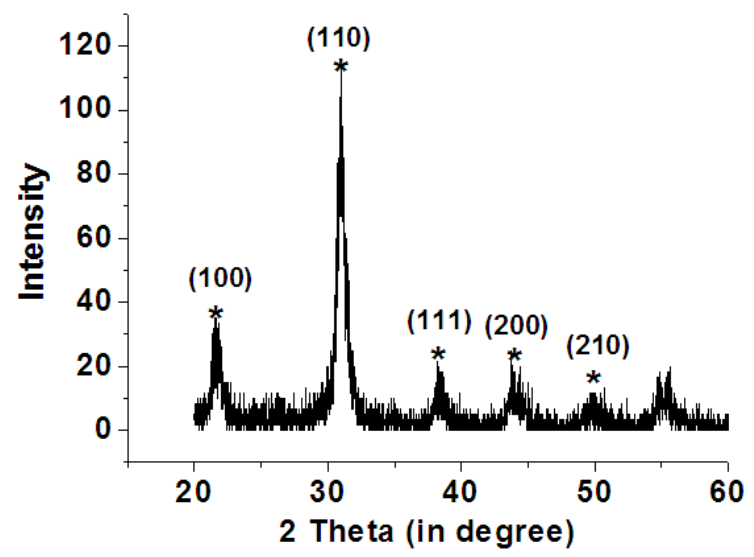

\subsubsection{Effect of Piezoelectric Material Flexibility on Electrical Performances of Tactile Sensors}

Our sensor consists of a piezoelectric material and a stainless steel substrate. Therefore the sensor flexibility is dominated by the properties of the stainless steel substrate material, as the Young's modulus of stainless steel is much higher than the PZT or P(VDF-TrFE) material, as shown in Table 1. Silicon wafers have been commonly used for the sensor substrate but the low Poisson's ratio limits the substrate flexibility.

Table 1. Material properties of PZT, PVDF, silicon and stainless steel.

\begin{tabular}{cccc}
\hline & Young's Modulus $(\mathbf{G P a})$ & Poisson's Ratio & Density $\left(\mathbf{k g} / \mathbf{m}^{\mathbf{3}}\right)$ \\
\hline PZT [41] & 70 & 0.3 & 7,500 \\
PVDF [42,43] & $2 \sim 4$ & 0.34 & 1.78 \\
Silicon [44] & 168.9 & 0.182 & 2,330 \\
Stainless steel [41] & 193 & 0.318 & 8,000 \\
\hline
\end{tabular}

To study the flexibility of the piezoelectric materials, it was reported that the electrical properties (such as J-E curve) of the flexible sensors under different bending were compared [39,40]. To compare the electrical properties of our piezoelectric material on the stainless steel substrate under various bending situations, we first performed the spin coating of the PZT material on the stainless steel substrate. As show in Figure 6(a,b), the PZT tactile sensor was fixed on the iron bars with different radius followed by the measurement of the material J-E curve of the sensor. From the measurement results, as shown in Figure 6(c), the material J-E curves of our PZT sensor secured at different iron bars showed only minor differences. These bending experiments created more curvature of the sensor 
than the real situations of the human pulse measurement. Therefore, it was demonstrated that our sensor can be utilized for human body measurement with various topologies by using our PZT material on the stainless steel substrate.

Figure 6. (a) Iron bars with different radius, (b) test set-up of our sensor flexibility testing, (c) J-E curves of our PZT sensor mounted without and with iron bars at different radius of R1-R3.
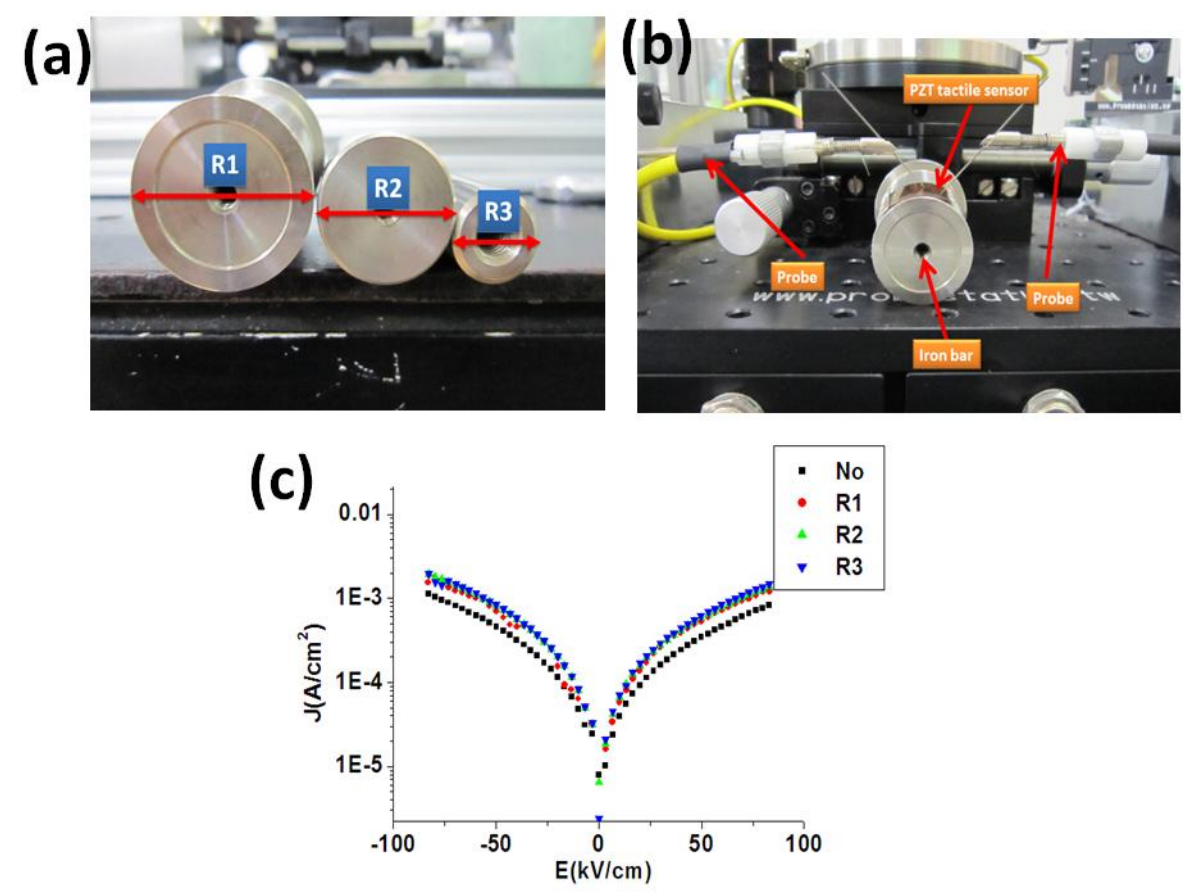

\subsection{Pulse Measurements on Various Human Body Regions and the Packaged PZT Thin Film Tactile} Sensor on Stainless Steel Dangerous Testing

\subsubsection{Monitoring of Human Body Pulse Waves}

For the human pulse measurement, it is important to use the flexible substrate to accommodate the various surface topologies of the human body. The flexible stainless steel substrate was selected as it was compatible with the fabrication of our targeted piezoelectric material and was more robust than other type of flexible (such as Al foil or polyimide). We also demonstrated a more diverse measurement (six arterial regions of the human body) than other research groups as our senor was able to detect most arterial regions of the human body weather it was close or far away from the human skin surface. The comparisons of our sensor and others were summarized in Table 2.

As shown in Figure 7(a), the general human body pulse waveform (thick aqua line) has two peaks by a human heart: the first peak (P position in the blue line waveform) from the heart systolic pressure and the second peak ( $\mathrm{D}$ position in the black line) from the diastolic pressure. The period and magnitude of the human body pulse waveform are constant under a resting condition. These repetitive signals were measured using the proposed tactile sensors at various regions of the human body, including the carotid artery, the brachial artery, the radial artery, the finger, the ankle artery, and the apical region, as show in Figure 7(b). 
Table 2. Comparison of piezo-electric tactile sensors with different flexible substrates.

\begin{tabular}{ccc}
\hline & Substrate & Application \\
\hline PVDF [23] & NA & Radial \\
PVDF [25] & NA & Brachial, Radial \\
AlN [21] & Polyimide $(8.5 \mu \mathrm{m})$ & Finger \\
AlN [22] & Aluminum foils $(1 \mu \mathrm{m}, 16 \mu \mathrm{m})$ & Femoral \\
PZ [45] & NA & Rubber tube \\
PZ [46] & NA & Elastic tube \\
PZT (this work) & Stainless steel & Carotid, Brachial, Finger, Ankle, Radial, Apical \\
\hline
\end{tabular}

Figure 7. (a)The heart systolic to diastolic and to produce the general human pulse waveform (b) the PZT tactile sensor to measure the human body artery pulse region.

\section{Heart systolic}

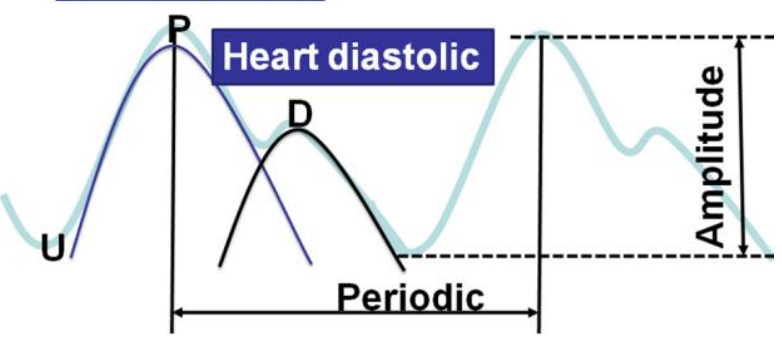

(a)

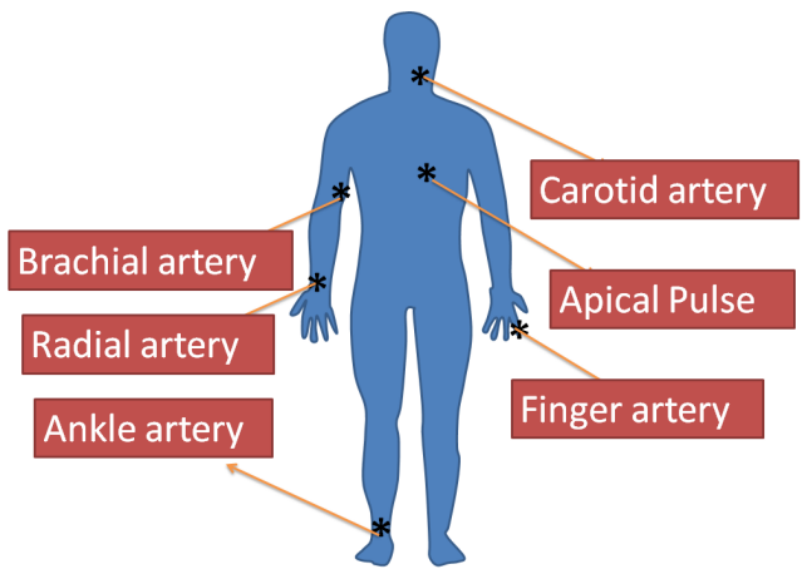

(b)

However, the geometry and shape of the human body is typically irregular and complex. For example, the topology of the carotid region is smoother compared to that of the finger region. In addition, the amplitude of the pulse signal is highly dependent on the distance between the surface of the skin and the artery underneath the skin. Therefore, the flexible tactile sensor was designed to be adapted to various human body regions and sense various amplitudes of pulses. As shown in Figure 8, the PZT tactile sensors sense the pulses of various human body regions from the carotid artery close to the human head to the ankle artery in the feet. Pulses from the carotid artery were first measured, and a large signal was obtained because of the smooth surface of the detected region. Next, the apical pulse from the heart was sensed for the heartbeat, and the waveform differed from that of the pulse monitored from other regions. In addition, weaker pulses from the finger and radial artery regions were 
observed compared to the stronger signals in the brachial artery region. However, in the ankle region, the pulse magnitude was large because the ankle artery is close to the surface of the skin. Using the proposed PZT-based flexible tactile sensors, various amplitudes and waveforms can be successfully detected at various areas of the human body, as shown in Figure 9.

Figure 8. A PZT flexible tactile sensor to sense different human body region (a) carotid artery (b) apical pulse (c) brachial artery (d) radical artery (e) finger artery (f) ankle artery region.

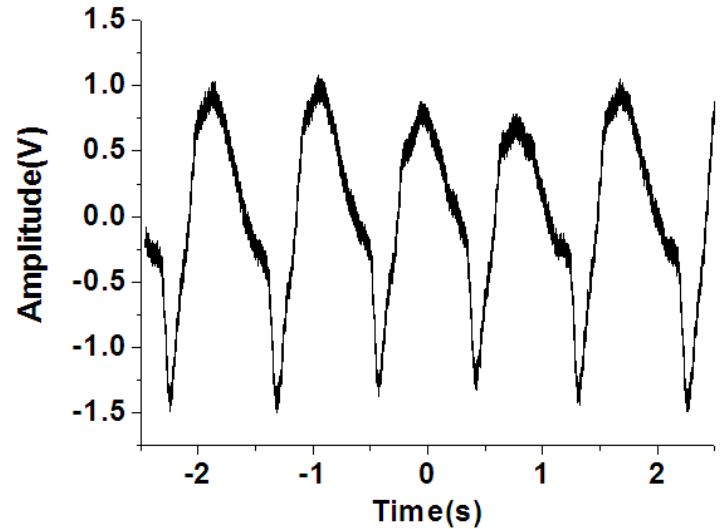

(a)

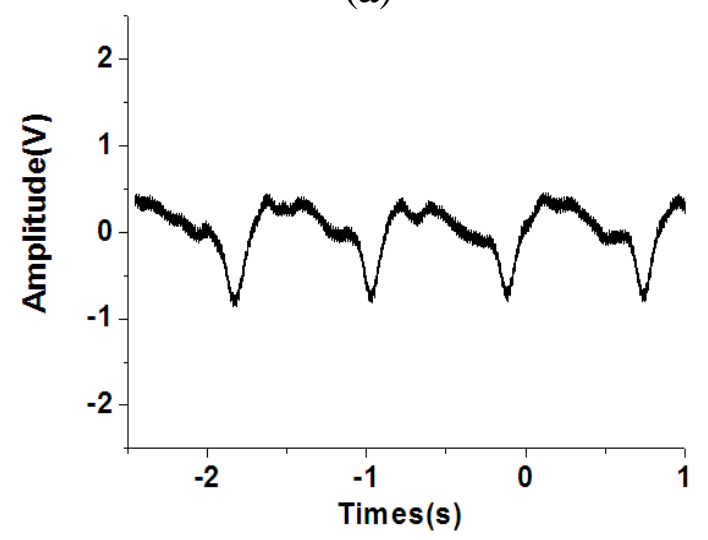

(c)

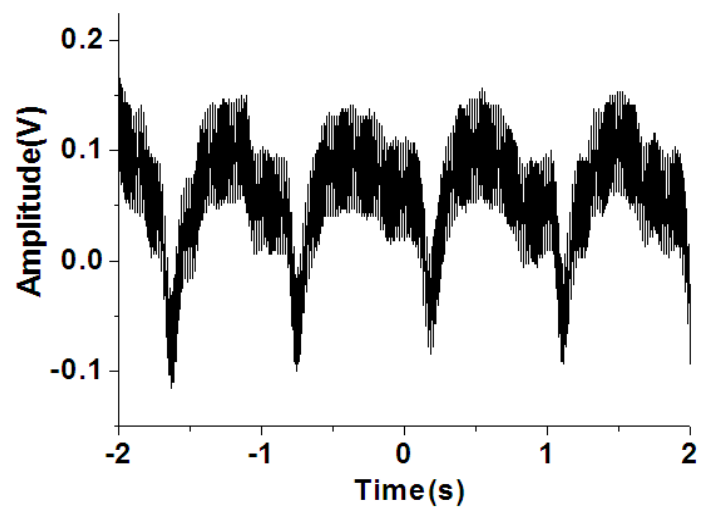

(e)

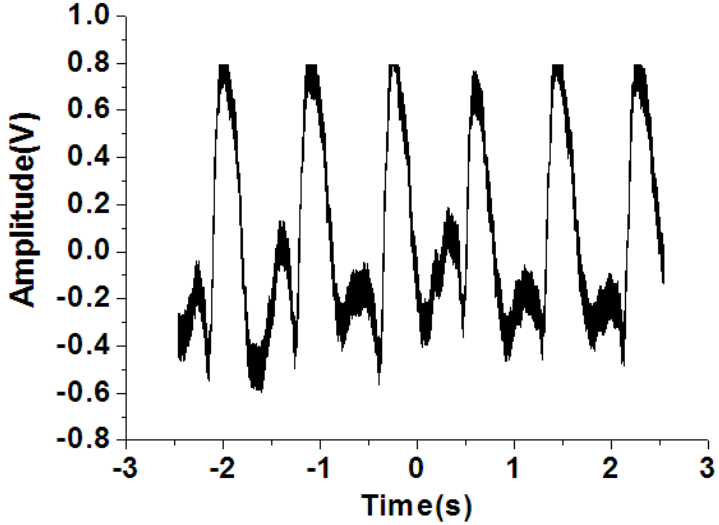

(b)

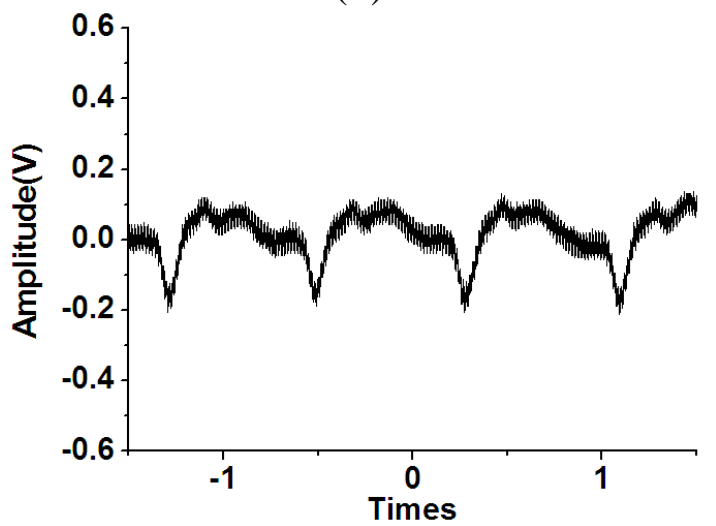

(d)

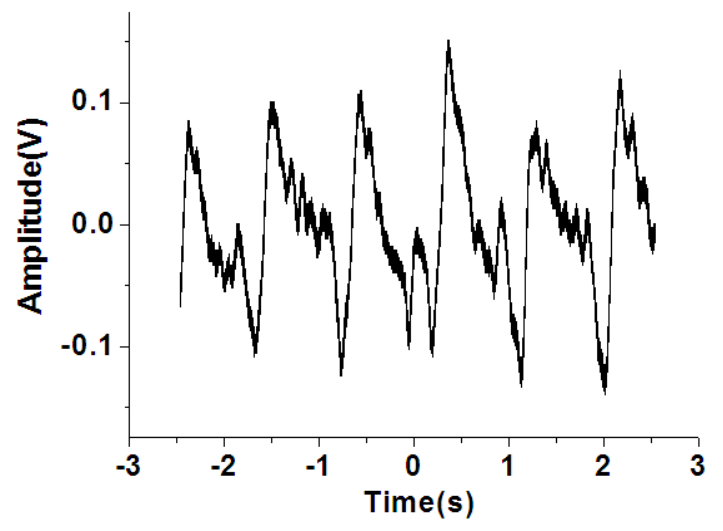

(f) 
Figure 9. Comparison of pulse amplitude and waveform measured from numerous regions of the human body with our sensor.

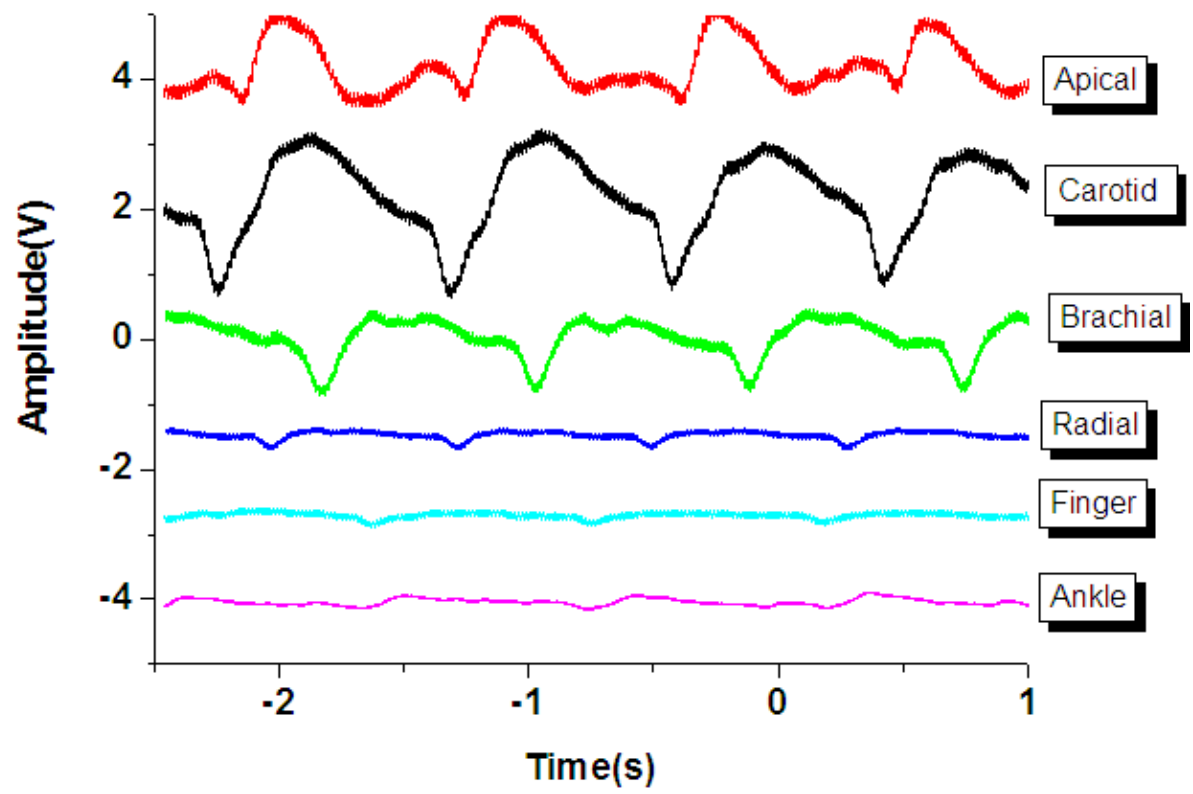

\subsubsection{Estimate of Human Body Pulse Wave Velocity (PWV)}

For the pulse wave velocity (PWV) measurement, the definition of the distance from apical to radial, brachial to radial and radial to ankle distance was shown in Figure 10(a) [47]. The distance from apical to radial artery is defined as "Distance $=4+5+6+7$ ", the distance from brachial to radial artery is defined as "Distance $=7$ ", and the distance from radial to ankle artery is defined as "Distance $=1+2+3-4-5-6-7$ ". The distance was measured with a standard ruler and the actual distance will be varied person by person. Therefore, when performing a real clinical test for a patient, it is important to utilize the correct distance in between each test spot to get accurate measurement. The human body PWV is a crucial index for patient health. As shown in Figure 10(b), the human body PWV can be estimated based on the time difference $\Delta \mathbf{t}$ of the two successive peaks of systolic pressure and diastolic pressure. We synchronized two measurements from differing regions of the human body and recorded the signals, as shown in Figure 10(c). The difference of the path length from the first region to the heart and the other region to the heart was D. Thus, the human body PWV was estimated based on the following formula:

$$
\mathrm{D} / \Delta t
$$

As shown in Table 2, the path distance to the heart between the apical artery and the radial artery is approximately $0.85 \mathrm{~m}$, and the time interval between the heart and radial artery is approximately $0.15 \mathrm{~s}$; therefore, the estimate of the PWV from the apical artery region to the radial artery region is $5.66 \mathrm{~m} / \mathrm{s}$. The same methodology can be applied to estimate the pulse wave velocities at various regions, as shown in Table 3. 
Figure 10. (a) PWV definition of the distance from apical to radial, brachial to radial and radial to ankle distance (b) The human body PWV calculation (c) the time interval between the apical to radial, brachial to radial and radial to ankle.

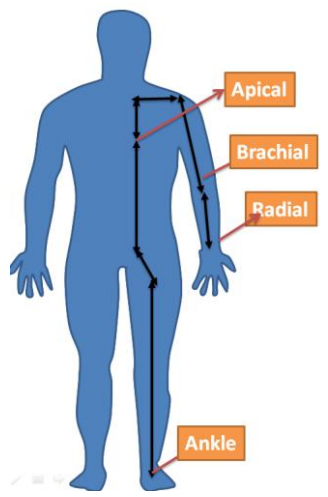

(a)

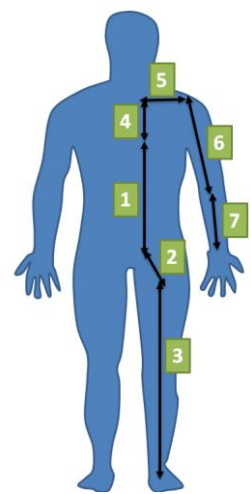

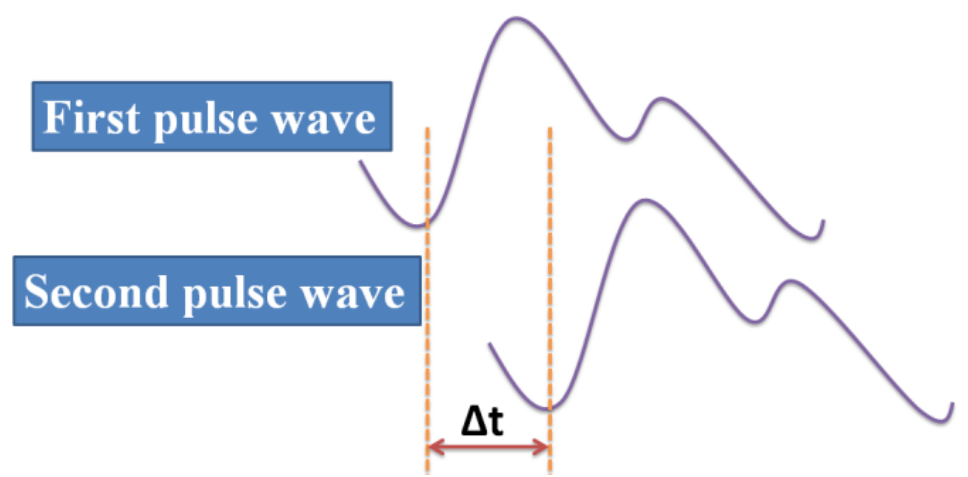

(b)
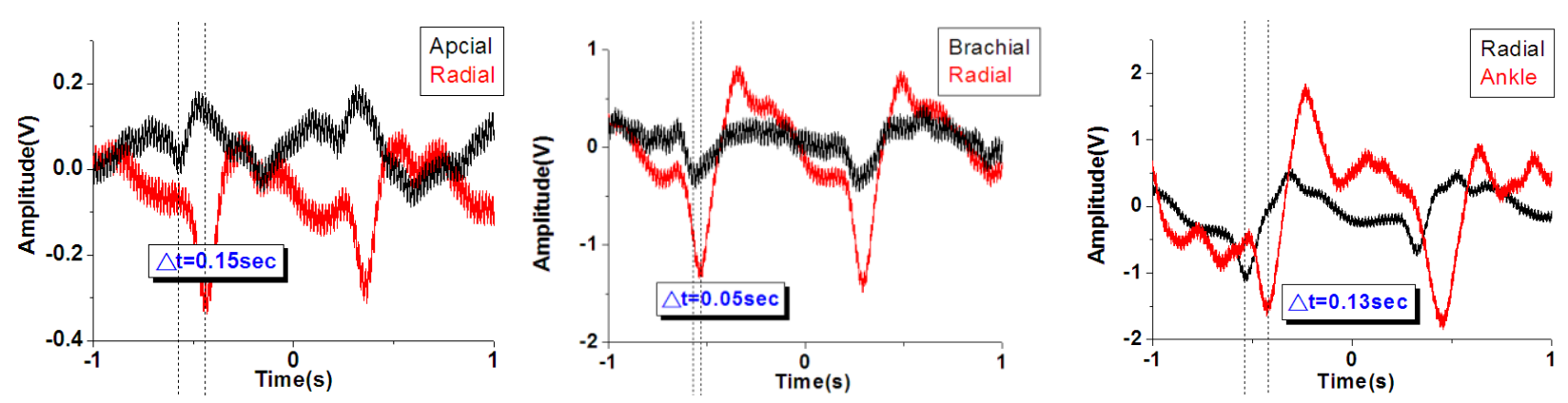

(c)

Table 3. The apical to radial, brachial to radial and radial to ankle human body (PWV).

\begin{tabular}{cccc}
\hline & Distance $(\mathbf{m})$ & $\triangle \mathbf{t}(\mathbf{s})$ & $\mathbf{P W V}(\mathbf{m} / \mathbf{s})$ \\
\hline apical to radial & 0.85 & 0.15 & 5.66 \\
brachial to radial & 0.2 & 0.05 & 4 \\
radial to ankle & 0.8 & 0.13 & 6.15 \\
\hline
\end{tabular}

\subsubsection{The Safety Tests of Packaged PZT Thin Film Tactile Sensor on Stainless Steel Substrate}

As shown in Figure 11(a-d), the packaged PZT thin film tactile sensor was touched and moved back and forth on the human skin. During the multiple tests (>100 times), no damages or scratches were found on the human skin. We also compared the safety of the unpackaged stainless steel plate and our packaged PZT tactile sensor. A scratch line was observed on a paper when touching and moving the unpackaged stainless steel plate over a paper while no scratches were observed when using our packaged PZT tactile sensor. From these safety tests, it is concluded that our packaged PZT thin film sensor with a stainless steel substrate is very safe for human pulse measurements. 
Figure 11. (a) The packaged PZT thin film sensor and the area to be touched, (b) the packaged tactile sensor touched the human skin, (c) the packaged tactile sensor was moved from left to right, (d) no scratches were found on the areas the sensor touched.

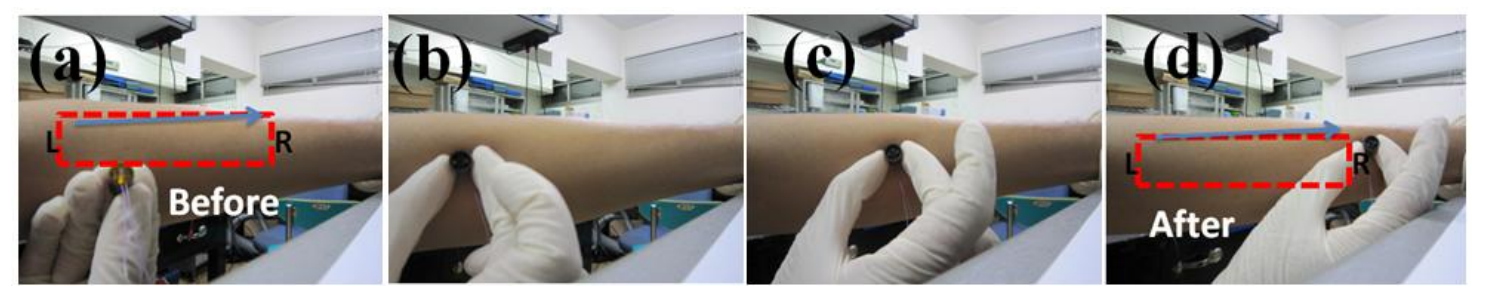

\section{Conclusions}

We have successfully fabricated PZT flexible tactile sensors using a sol-gel deposition process. The PZT thin-film was deposited on the flexible stainless steel substrate to enable broad pulse detection capability for various human body regions, such as the carotid region, the brachial region, the finger, the ankle artery, the radial artery, and the apical region. The PZT flexible tactile sensor is sensitive to several human body regions with various geometries. The amplitude of the human pulse waveform is large on the carotid artery, whereas that the finger region is small. Using the proposed PZT-based flexible tactile sensors, a wide range of pulse sensing from numerous regions of the human body can be achieved for the monitoring human health. Finally, human body pulse wave velocities from the apical pulse region to the carotid region, the brachial artery region, the finger, and the ankle artery of the human body were estimated and the results were consistent.

\section{Acknowledgments}

The authors are grateful to the funding support from Nation Science Council, Taiwan under contract number NSC-101-2220-E-002-002-.

\section{References}

1. Aoyagi, S.; Matsuda, T.; Kong, T.-W.; Ishimaru, T.; Suzuki, M.; Inoue, K. Proposal and development of arrayed sole sensor for legged robot and contact force detection using neural networks. IEEE Sens. J. 2011, 11, 2048-2056.

2. Chang, W.-Y.; Fang, T.-H.; Shen, Y.-T.; Lin, Y.-C. Flexible electronics sensors for tactile multiscanning. Rev. Sci. Instrum. 2009, 80, 084701.

3. Hao, X.; Jiang, Y.; Takao, H.; Maenaka, K.; Higuchi, K. An annular mechanical temperature compensation structure for gas-sealed capacitive pressure sensor. Sensors 2012, 12, 8026-8038.

4. Chen, Z.H.; Luo, R.C. Design and implementation of capacitive proximity sensor using microelectromechanical systems technology. IEEE Trans. Ind. Electron. 1998, 45, 886-894.

5. Cheng, M.Y.; Huang, X.H.; Ma, C.W.; Yang, Y.J. A flexible capacitive tactile sensing array with floating electrodes. J. Micromech. Microeng. 2009, 19, 115001.

6. Vidal-Verdú, F.; Oballe-Peinado, Ó.; Sánchez-Durán, J.A.; Castellanos-Ramos, J.; Navas-González, R. Three realizations and comparison of hardware for piezoresistive tactile sensors. Sensors 2011, 11, 3249-3266. 
7. Gutierrez, C.A.; Meng, E.J. Impedance-based force transduction within fluid-filled parylene microstructures. Microelectromech. Syst. 2011, 20, 1098-1108.

8. Park, C.-S.; Park, J.; Lee, D.-W. A piezoresistive tactile sensor based on carbon fibers and polymer substrates. Microelectron. Eng. 2009, 86, 1250-1253.

9. Charlot, B.; Parrain, F.; Galy, N.; Basrour, S.; Courtois, B.J. A sweeping mode integrated fingerprint sensor with 256 tactile microbeams. Microelectromech. Syst. 2004, 13, 636-644.

10. Gutierrez, C.A.; Meng, E. Parylene-based electrochemical-MEMS transducers. J. Microelectromech. Syst. 2010, 19, 1352-1361.

11. Bakke, T.; Vogl, A.; Zero, O.; Tyholdt, F.; Johansen, I.R.; Wang, D. A novel ultra-planar, long-stroke and low-voltage piezoelectric micromirror. J. Micromech. Microeng. 2010, 20, 064010.

12. Lee, C.; Hsiao, F.L.; Kobayashi, T.; Koh, K.H.; Ramana, P.V.; Xiang, W.F.; Yang, B.; Tan, C.W.; Pinjala, D. A 1-V operated MEMS variable optical attenuator using piezoelectric PZT thin-film actuators. IEEE J. Sel. Top. Quantum Electron. 2009, 15, 1529-1536.

13. Guerre, R.; Drechsler, U.; Bhattacharyya, D.; Rantakari, P.; Stutz, R.; Wright, R.V.; Milosavljevic, Z.D.; Vaha-Heikkila, T.; Kirby, P.B.; Despont, M. Wafer-level transfer technologies for PZT-based RF MEMS switches. J. Microelectromech. Syst. 2010, 19, 548-560.

14. Ko, F.H.; Hsu, Y.C.; Wang, M.T.; Huang, G.W.S. Fabrication of a gas sensor with a piezoelectric PZT film deposited by a novel hydrothermal microwave-assisted annealing. Microelectron. Eng. 2007, 84, 1300-1304.

15. Kohli, M.; Wuethrich, C.; Brooks, K.; Willing, B.; Forster, M.; Muralt, P.; Setter, N.; Ryser, P. Pyroelectric thin-film sensor array. Sens. Actuators A Phys. 1997, 60, 147-153.

16. Liu, W.G.; Ko, J.S.; Zhu, W.G. Influences of thin Ni layer on the electrical and absorption properties of PZT thin film pyroelectric IR sensors. Infrared Phys. Technol. 2000, 41, 169-173.

17. Choi, W.J.; Jeon, Y.; Jeong, J.H.; Sood, R.; Kim, S.G. Energy harvesting MEMS device based on thin film piezoelectric cantilevers. J. Electroceram. 2006, 17, 543-548.

18. Jeon, Y.B.; Sood, R.; Jeong, J.H.; Kim, S.G. MEMS power generator with transverse mode thin film PZT. Sens. Actuators A Phys. 2005, 122, 16-22.

19. Lee, B.S.; Lin, S.C.; Wu, W.J.; Wang, X.Y.; Chang, P.Z.; Lee, C.K. Piezoelectric MEMS generators fabricated with an aerosol deposition PZT thin film. J. Micromech. Microeng. 2009, 19, 065014.

20. Dahiya, R.S.; Cattin, D.; Adami, A.; Collini, C.; Barboni, L.; Valle, M.; Lorenzelli, L.; Oboe, R.; Metta, G.; Brunetti, F. Towards tactile sensing system on chip for robotic applications. IEEE Sens. J. 2011, 11, 3216-3226.

21. Akiyama, M.; Morofuji, Y.; Kamohara, T.; Nishikubo, K.; Ooishi, Y.; Tsubai, M.; Fukuda, O.; Ueno, N. Preparation of oriented aluminum nitride thin films on polyimide films and piezoelectric response with high thermal stability and flexibility. Adv. Funct. Mater. 2007, 17, 458-462.

22. Akiyama, M.; Ueno, N.; Nonaka, K.; Tateyama, H. Flexible pulse-wave sensors from oriented aluminum nitride nanocolumns. Appl. Phys. Lett. 2003, 82, 1977.

23. Clemente, F.; Arpaia, P.; Cimmino, P. A piezo-film-based measurement system for global haemodynamic assessment. Physiol. Meas. 2010, 31, 697-714. 
24. Gonzalez-Moran, C.O.; Flores-Cuautle, J.J.A.; Suaste-Gomez, E. A piezoelectric plethysmograph sensor based on a Pt wire implanted lead lanthanum zirconate titanate bulk ceramic. Sensors 2010, 10, 7146-7156.

25. McLaughlin, J.; McNeill, M.; Braun, B.; McCormack, P.D. Piezoelectric sensor determination of arterial. Physiol. Meas. 2003, 24, 693-702.

26. Kyriacou, P.A.; Shafqat, K.; Pal, S.K. Pilot investigation of photoplethysmographic signals and blood oxygen saturation values during blood pressure cuff-induced hypoperfusion. Measurement 2009, 42, 1001-1005.

27. Spigulis, J. Optical noninvasive monitoring of skin blood pulsations. Appl. Opt. 2005, 44, 1850-1857.

28. Teng, X.F.; Zhang, Y.T. The effect of contacting force on photoplethysmographic signals. Physiol. Meas. 2004, 25, 1323-1335.

29. Phillips, J.P.; Hickey, M.; Kyriacou, P.A. Evaluation of electrical and optical plethysmography sensors for noninvasive monitoring of hemoglobin concentration. Sensors 2012, 12, 1816-1826.

30. Bernjak, A.; Stefanovska, A. Pulse transit times to the capillary bed evaluated by laser doppler flowmetry. Physiol. Meas. 2009, 30, 245-260.

31. Ayres, J.W.; Lalande, F.; Chaudhry, Z.; Rogers, C.A. Qualitative impedance-based health monitoring of civil infrastructures. Smart Mater. Struct. 1998, 7, 599-605.

32. Annamdas, V.G.M.; Soh, C.K. Application of electromechanical impedance technique for engineering structures: Review and future issues. J. Intell. Mater. Syst. Struct. 2010, 21, 41-59.

33. Qi, Y.; Jafferis, N.T.; Lyons, K.; Lee, C.M.; Ahmad, H.; McAlpine, M.C. Piezoelectric ribbons printed onto rubber for flexible energy conversion. Nano Lett. 2010, 10, 524-528.

34. Chang, W.Y.; Fang, T.H.; Lin, H.J.; Shen, Y.T.; Lin, Y.C. A large area flexible array sensors using screen printing technology. J. Disp. Technol. 2009, 5, 178-183.

35. Choi, D.; Lee, K.Y.; Lee, K.H.; Kim, E.S.; Kim, T.S.; Lee, S.Y.; Kim, S.W.; Choi, J.Y.; Kim, J.M. Piezoelectric touch-sensitive flexible hybrid energy harvesting nanoarchitectures. Nanotechnology 2010, 21, 405503.

36. Mannsfeld, S.C.B.; Tee, B.C.K.; Stoltenberg, R.M.; Chen, C.; Barman, S.; Muir, B.V.O.; Sokolov, A.N.; Reese, C.; Bao, Z.N. Highly sensitive flexible pressure sensors with microstructured rubber dielectric layers. Nat. Mater. 2010, 9, 859-864.

37. Sato, N.; Shigematsu, S.; Morimura, H.; Yano, M.; Kudou, K.; Kamei, T.; Machida, K. Novel surface structure and its fabrication process for MEMS fingerprint sensor. IEEE Trans. Electron. Dev. 2005, 52, 1026-1032.

38. Yu, S.; Wong, T.K.S.; Hu, X.; Pita, K. Sol-gel derived mesoporous silica films used as low dielectric constant materials. Thin Solid Films 2004, 462-463, 311-315.

39. Lee, S.; Kim, H.; Yun, D.J.; Rhee, S.W.; Yong, K. Resistive switching characteristics of ZnO thin film grown on stainless steel for flexible nonvolatile memory devices. Appl. Phys. Lett. 2009, 95, 262113.

40. Yun, D.-J.; Lim, S.-H.; Lee, T.-W.; Rhee, S.-W. Fabrication of the flexible pentacene thin-film transistors on 304 and 430 stainless steel (SS) substrate. Org. Electron. 2009, 10, 970-977.

41. Suzuki, T.; Kanno, I.; Loverich, J.J.; Kotera, H.; Wasa, K. Characterization of Pb(Zr,Ti)O-3 thin films deposited on stainless steel substrates by RF-magnetron sputtering for MEMS applications. Sens. Actuators A Phys. 2006, 125, 382-386. 
42. Ali, R.; Mahapatra, D.R.; Gopalakrishnan, S. An analytical model of constrained piezoelectric thin film sensors. Sens. Actuators A Phys. 2004, 116, 424-437.

43. Caneva, C.; De Rosa, I.M.; Sarasini, F. Monitoring of impacted aramid-reinforced composites by embedded PVDF acoustic emission sensors. Strain 2008, 44, 308-316.

44. Deshpande, M.; Saggere, L. PZT thin films for low voltage actuation: Fabrication and characterization of the transverse piezoelectric coefficient Sens. Actuators A Phys. 2007, 135, 690-699.

45. Pereira, H.C.; Pereira, T.; Almeida, V.; Borges, E.; Figueiras, E.; Simoes, J.B.; Malaquias, J.L.; Cardoso, J.M.R.; Correia, C.M.B. Characterization of a double probe for local assessment Physiol. Meas. 2010, 31, 1449-1465.

46. Almeida, V.G.; Pereira, H.C.; Pereira, T.; Figueiras, E.; Borges, E.; Cardoso, J.M.R.; Correia, C. Piezoelectric probe for pressure waveform estimation in flexible tubes and its application to the cardiovascular system. Sens. Actuators A Phys. 2011, 169, 217-226.

47. Padilla, J.M.; Berjano, E.J.; Saiz, J.; Rodriguez, R.; Facila, L. Pulse wave velocity and digital volume pulse as indirect estimators of blood pressure: Pilot study on healthy volunteers. Cardiovasc. Eng. 2009, 9, 104-112.

(C) 2013 by the authors; licensee MDPI, Basel, Switzerland. This article is an open access article distributed under the terms and conditions of the Creative Commons Attribution license (http://creativecommons.org/licenses/by/3.0/). 